\title{
Quantification Of Actinides By EPMA: a New Accurate Standardless Approach
}

\author{
A. $\mathrm{Moy}^{1,2}$, C. Merlet ${ }^{1}$, O. Dugne ${ }^{2}$. \\ 1. GM, CNRS, Université de Montpellier II, Place E. Bataillon, 34095 Montpellier, France. \\ 2. CEA, DEN, DTEC, SGCS, LMAC, 30207 Bagnols-sur-Cèze, France.
}

Electron probe microanalysis (EPMA) is used to quantify with a high accuracy the amount of different elements present on a sample of unknown composition. In the nuclear field, EPMA is largely used to quantify the amount of actinides present in fresh and irradiated fuels. EPMA is also used to manage waste disposal, to analyze sample from severe accidents and to assess the design of next-generation fuels. However, quantitative EPMA is not always possible to achieve for these materials due to the lack of suitable reference standards for the radionuclides (e.g. americium or curium). To overcome this difficulty, standardless methods of analysis are employed with mean of virtual calculated standards. These calculated standards are generally obtained from empirical formulae based on experimental extrapolations or from theoretical calculations that require physical parameters which are poorly known as it is the case for the $\mathrm{x}$-ray production cross section.

In the present work, L- and M-shell absolute $\mathrm{x}$-ray production cross sections were determined experimentally for elements $\mathrm{Pb}(Z=82)[1-2]$, $\mathrm{Th}(Z=90)$ and $\mathrm{U}(Z=92)$ [2-3] by electron impact using ultrathin self-supporting targets $(0.2-8 \mathrm{~nm})[4,5]$. The measured cross sections have been compared, with the distorted-wave Born approximation (DWBA) calculated by Bote et al. [6] and with the predictions of analytical formulae widely used in practical applications. For the conversion of innershell ionization cross sections into x-ray production cross sections, atomic relaxation parameters were extracted from the Evaluated Atomic Data Library (EADL). Our measured x-ray production cross sections agree satisfactorily, within the uncertainties of the measurements and of the relaxation parameters, with the predictions of the DWBA calculations (Fig.1). This confirms the usefulness of Bote et al.'s predictive formula for the calculation of virtual standards in the EPMA analysis of actinide elements. These ionization cross sections were used into the Monte Carlo simulation code PENELOPE [7] to calculate the X-ray intensity produced by pure actinide standards (Fig.2). The X-ray intensities were calculated for elements with atomic number $89 \leq Z \leq 99$ and for accelerating voltage ranging from the ionization threshold up to $40 \mathrm{kV}$ with a step of $0.5 \mathrm{kV}$. For a practical use, the calculated intensities for the most intense L and M lines were stored in a database in function of the accelerating voltage.

Practically, to be used as a calculated standard in a WDS microprobe, calculated intensity has to take into account the characteristics of the spectrometer used for the measurement. Indeed, the x-ray intensity emitted from a sample or calculated by simulations and the recorded signal are related by the efficiency of the spectrometer and by the spectral broadening of the x-ray line.

- The spectrometer efficiency, which is function of the measured photon energy, can be obtained by comparing the bremsstrahlung intensity produced by a bulk standard (of Ni for the L lines, C, Al and Si for the M lines) with the bremsstrahlung calculated by the code PENELOPE at the same photon energy. For a convenient use, the emitted bremsstrahlung was calculated for 5, 10 and $20 \mathrm{kV}$ accelerating voltage and stored in a database. The spectrometer efficiency at the energy of interest is then given by the ratio of the measured bremsstrahlung by the calculated bremsstrahlung.

- The spectral broadening is taken into account by recording a high resolution spectrum of the measured line and by calculating the associated area with a set of pseudo-Voigt functions. 
The virtual calculated intensity standard of the x-ray line of interest is finally obtained by calculating the ratio between the calculated x-ray intensity and the normalized area of the x-ray line timed by the spectrometer efficiency. An easy to use program interface was developed to simplify and automate the calculations of the virtual calculated intensity standard and to manage the calculated bremsstrahlung and $\mathrm{x}$-ray intensities. The program calculates the virtual intensity standard and stores it into the standards database of the microprobe. Standardless quantitative measurements of actinides are then possible to perform on an unknown sample as a simple quantification measurement.

The predictions of our calculated standards have been compared with the x-ray intensity of known composition actinide samples (such as $\mathrm{UO}_{2}, \mathrm{ThO}_{2}, \mathrm{ThF}_{4}$, and $\mathrm{PuO}_{2}$ ) and with the data acquired during previous measurement projects [8]. Actinide quantifications performed by virtual standards were found to be in fair agreement with the expected results. This confirms the reliability of the developed virtual standards and demonstrates that actinide quantification by EPMA can now be possible to perform at a good level of accuracy without material actinide standards.

\section{References:}

[1] A Moy et al, J. Phys. B: At. Mol. Opt. Phys. 46 (2013) 115202.

[2] A Moy et al, IEEE Trans. Nucl. Sci. (2014), accepted.

[3] A Moy et al, J. Phys. B: At. Mol. Opt. Phys. 47 (2014) 055202.

[4] C Merlet et al, Phys. Rev. A 78 (2008) 022704.

[5] C Merlet et al, Phys. Rev. A 73 (2006) 062719.

[6] D Bote et al, At. Data and Nucl. Data Tables 96 (2009) p.871.

[7] F Salvat et al, "PENELOPE-2012: A Code System for Monte Carlo Simulation of Electron and Photon Transport", (OECD/NEA Data Bank, Issy-les-Moulineaux, France).

[8] C Merlet et al, Microsc. Microanal. 14 (Suppl 2) (2008) p.1094.

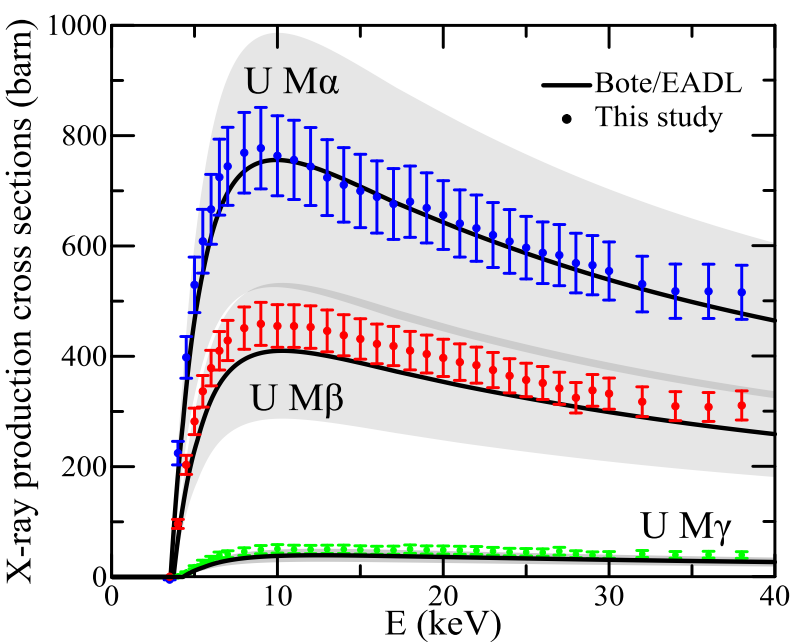

Figure 1. Experimental and theoretical predictions of the $\mathrm{M}$ x-ray line production cross sections of $U$.

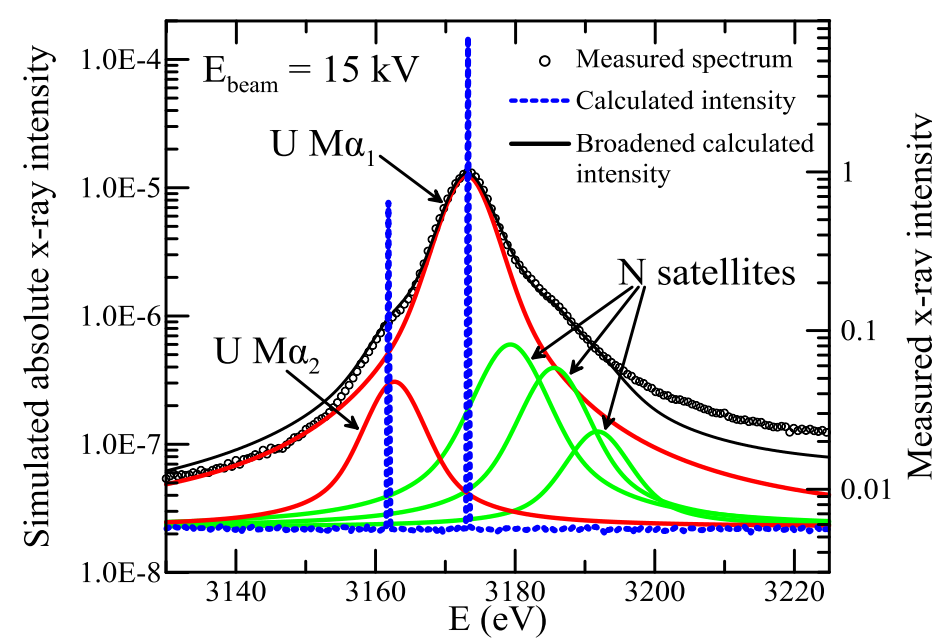

Figure 2. Calculated intensity and broadened calculated intensity of the $\mathrm{U} \mathrm{M} \alpha$ lines with an electron beam of $15 \mathrm{kV}$. 